\title{
Exigências térmicas de Doru lineare Eschs. e Doru luteipes Scudder em laboratório
}

\author{
Thermal requirements of Doru lineare Eschs. and Doru luteipes Scudder in the laboratory
}

\author{
Amarildo Pasini' ${ }^{I}$ osé Roberto Postali Parra ${ }^{\mathrm{II}}$ Dori Edson Nava ${ }^{\mathrm{III}}$ Alessandra Regina Butnariu ${ }^{\mathrm{IV}}$
}

\section{RESUMO}

\begin{abstract}
Algumas espécies de tesourinhas (Dermaptera: Forficulidae) são importantes predadoras da lagarta-docartucho do milho Spodoptera frugiperda (J.E. Smith) (Lepidoptera: Noctuidae). Neste trabalho, avaliou-se o efeito de temperaturas constantes no desenvolvimento de Doru luteipes (Scudder) e Doru lineare (Eschs.). D. luteipes não completou seu desenvolvimento nas temperaturas de 30 e $32^{\circ} \mathrm{C}$, enquanto $\boldsymbol{D}$. lineare não completou apenas na temperatura de $32^{\circ} \mathrm{C}$. O período de incubação variou de $8,0\left(25^{\circ} \mathrm{C}\right)$ a 17,2 dias $\left(18^{\circ} \mathrm{C}\right)$ e de $4,6\left(30^{\circ} \mathrm{C}\right)$ a 14,5 dias $\left(18^{\circ} \mathrm{C}\right)$, para $\boldsymbol{D}$. luteipes e $\boldsymbol{D}$. lineare, respectivamente. A duração do período ninfal variou de 30,4 dias $\left(25^{\circ} \mathrm{C}\right)$ a 63,6 dias $\left(18^{\circ} \mathrm{C}\right)$, para $\boldsymbol{D}$. luteipes, e de 20,4 dias $\left(30^{\circ} \mathrm{C}\right)$ a 60,5 dias $\left(18^{\circ} \mathrm{C}\right)$, para D. lineare. A duração do ciclo biológico (ovo-adulto) variou de $38,4\left(25^{\circ} \mathrm{C}\right)$ a 80,8 dias $\left(18^{\circ} \mathrm{C}\right)$, para $\mathbf{D}$. luteipes, e de $25\left(30^{\circ} \mathrm{C}\right)$ a 75 dias $\left(18^{\circ} \mathrm{C}\right)$, para D. lineare. Portanto, as temperaturas elevadas foram desfavoráveis para ambas as espécies, observando-se que $\boldsymbol{D}$. luteipes é mais susceptível à elevação térmica.
\end{abstract}

Palavras-chave: constante térmica, controle biológico, grausdia, predador, tesourinha.

\section{ABSTRACT}

Some Earwigs species (Dermaptera: Forficulidae) are important predators of the fall armyworm, Spodoptera frugiperda (J.E. Smith) (Lepidoptera: Noctuidae). The effects of constant temperatures on the development of Doru luteipes (Scudder) and Doru lineare (Eschs.) were evaluated. The development of $\boldsymbol{D}$. luteipes was not completed at 30 and $32^{\circ} \mathrm{C}$, whereas $\boldsymbol{D}$. lineare did not complete only at $32^{\circ} \mathrm{C}$. The incubation period ranged from $8.0\left(25^{\circ} \mathrm{C}\right)$ to 17.2 days $\left(18^{\circ} \mathrm{C}\right)$ for $\boldsymbol{D}$.

\begin{abstract}
luteipes and $\boldsymbol{D}$. lineare, respectively. The duration of the nymphal stage ranged from $30.4\left(25^{\circ} \mathrm{C}\right)$ to 63.6 days $\left(18^{\circ} \mathrm{C}\right)$ for $\boldsymbol{D}$. luteipes and, from $20.4\left(30^{\circ} \mathrm{C}\right)$ to 60.5 days $\left(18^{\circ} \mathrm{C}\right)$ for $\boldsymbol{D}$. lineare. The duration of the biological cycle (egg-adult) varied from $38.4\left(25^{\circ} \mathrm{C}\right)$ to 80.8 days $\left(18^{\circ} \mathrm{C}\right)$ for $\boldsymbol{D}$. luteipes and from $25\left(30^{\circ} \mathrm{C}\right)$ to 75 days $\left(18^{\circ} \mathrm{C}\right)$ for $\boldsymbol{D}$. lineare. Therefore, high temperatures were unfavorable for both species, but D. luteipes is more susceptible to higher temperatures.
\end{abstract}

Key words: biological control, degree-days, earwigs, predator, thermal constant.

\section{INTRODUÇÃO}

Diversas espécies de insetos danificam a cultura do milho desde a semeadura até a fase da formação dos grãos (VIANA, 2004). Entre as mais prejudiciais, encontra-se a lagarta-do-cartucho do milho Spodoptera frugiperda (J.E. Smith) (Lepidoptera: Noctuidae), cujo dano ocorre pela raspagem e perfuração das folhas já emitidas, bem como daquelas em formação no cartucho do milho, caracterizando o nome popular da espécie. Além disto, as lagartas podem ainda broquear a base da planta e atacar a espiga. Com o plantio do milho "safrinha”, o ataque se intensificou, e as perdas oscilam entre $34 \mathrm{a} 40 \%$ (FERNANDES, 2003).

O controle da lagarta-do-cartucho tem sido realizado basicamente por agrotóxicos, mas algumas

'Departamento de Agronomia, Centro de Ciências Agrárias, Universidade Estadual de Londrina (UEL), CP 6001, 86051-990,

Londrina, PR, Brasil. E-mail: pasini@uel.br. Autor para correspondência.

"Departamento de Entomologia, Fitopatologia e Zoologia Agrícola, Escola Superior de Agricultura "Luiz de Queiroz" (ESALQ),

Universidade de São Paulo (USP), Piracicaba, SP, Brasil.

IIIEmbrapa Clima Temperado, Pelotas, RS, Brasil.

${ }^{\mathrm{IV}}$ Departamento de Ciências Biológicas, Universidade do Estado de Mato Grosso (UFMT), Tangará da Serra, MT, Brasil. 
espécies da ordem Dermaptera, conhecidas como “tesourinhas”, são relatadas como importantes predadoras da referida praga. Entre essas predadoras, a espécie Doru lineare (Eschs.) pode ser mencionada, por ser frequente na cultura do milho (ROMEROSUELDO \& CUEZZO, 2001). Também são registradas como entomófagos nas culturas da soja (LEITE \& LARA, 1985), do algodão (SOARES et al., 1995; SOARES \& BUSOLI, 2000) e do trigo (GASSEN,1986). Além desta, Doru luteipes (Scudder), também faz parte do complexo de inimigos naturais de $\boldsymbol{S}$. frugiperda (REIS et al., 1988; CRUZ, 1991; CRUZ, 1994; GUERREIRO et al., 2003; PICANÇO et al., 2003; FIGUEIREDO et al., 2006), de Helicoverpa zea (Boddie) (Lepidoptera: Noctuidae) (CRUZ et al., 1995) e de Ascia monustes orseis (Goudart) (Lepidoptera: Pieridae) (PICANÇO et al., 2003).

CRUZ (2007) menciona que $\boldsymbol{D}$. luteipes é um dos inimigos naturais mais importantes na supressão de pragas na cultura do milho, pois as ninfas podem consumir diariamente cerca de 10 ovos e/ou lagartas de $\boldsymbol{S}$. frugiperda, e os adultos podem consumir diariamente 20 lagartas de primeiro e segundo ínstar. Além de $\boldsymbol{S}$. frugiperda, $\boldsymbol{D}$. luteipes preda ovos e lagartas de $\boldsymbol{H}$. zea (CRUZ, 2007), assim como ninfas e adultos do pulgão-verde do sorgo (Schizaphis graminum (Rondani) (Hemiptera: Aphididae)) (ALVARENGA et al., 1995a,b; ALVARENGA et al., 1996; CRUZ, 2007).

Devido à relevância dessa espécie como agente de mortalidade biótica, estudos visando ao controle biológico por conservação, com a utilização de inseticidas seletivos, têm sido realizados (SIMÕES et al.,1998; PICANÇO et al., 2003). Entretanto, ainda que se reconheça a importância dos predadores na redução populacional de insetos-praga, dermápteros não vem sendo utilizados em programas de controle biológico aplicado, devido, principalmente, à falta de conhecimento das suas características biológicas (CÔNSOLI \& PARRA, 1997; BERTI FILHO \& CIOCIOLA, 2002). PASINI et al. (2007) demonstraram a possibilidade da criação de $\boldsymbol{D}$. Iuteipes em laboratório; porém, há carência de estudos sobre a influência de fatores abióticos, como temperatura, no seu desenvolvimento. Segundo HAGEN et al. (1976), tal parâmetro é fundamental para o sucesso no uso de predadores em programas de controle biológico.

O efeito da temperatura sobre o ciclo de vida e a determinação das exigências térmicas dos inimigos naturais possibilitam prever e controlar a produção em laboratório, bem como estabelecer a temperatura ótima para o desenvolvimento e o sincronismo das criações do inseto-praga e do predador e estimar o número de gerações anuais ou durante o ciclo de produção das culturas (PARRA, 1997; PRATISSOLI \& PARRA, 2000; NAVA et al., 2005).

Os objetivos deste trabalho foram avaliar o efeito de temperaturas constantes no desenvolvimento dos predadores $\boldsymbol{D}$. luteipes e $\boldsymbol{D}$. lineare e determinar as exigências térmicas, visando à otimização da criação em laboratório, a fim de subsidiar futuras pesquisas acerca da utilização dessas espécies para o controle de S. frugiperda, entre outras espécies de insetos que atacam o milho.

\section{MATERIAL E MÉTODOS}

Os estudos foram conduzidos no Laboratório de Biologia de Insetos do Departamento de Entomologia e Acarologia da Escola Superior de Agricultura “Luiz de Queiroz” da Universidade de São Paulo, em Piracicaba, São Paulo (SP), com as espécies D. luteipes e D. lineare.

Para o estabelecimento da criação, coletaram-se, em lavouras de milho, em Londrina, Paraná (PR), adultos de $\boldsymbol{D}$. luteipes e $\boldsymbol{D}$. lineare que foram mantidos em laboratório, em recipientes plásticos transparentes com tampa $(5,0 \mathrm{~cm}$ de altura $\mathrm{x} 4,5 \mathrm{~cm}$ de diâmetro de base x 6,5cm de diâmetro superior), contendo em seu interior canudos plásticos transparentes $(0,5 \mathrm{~cm}$ de diâmetro $\mathrm{x} 6,0 \mathrm{~cm}$ de comprimento) e vedados em uma das extremidades com algodão umedecido, conforme metodologia descrita por PASINI et al. (2007). Autilização desse sistema garantiu abrigo aos insetos, uma vez que essas espécies são tigmotrópicas. Os adultos e as ninfas foram alimentados com pupas moídas do bicho-da-seda Bombyx mori (L.) (Lepidoptera: Bombycidae), associadas a grãos de pólen coletados por abelha, Apis mellifera (L.) (Hymenoptera: Apidae) (pólen apícola de flores silvestres desidratado). Esses alimentos foram oferecidos às tesourinhas em formas de papel laminado nำ. Para a criação de manutenção, foram realizados os seguintes procedimentos: limpeza dos recipientes, troca de alimento (farinha de bicho-da-seda e pólen) e umedecimento do algodão, duas vezes por semana.

Para estudar o efeito da temperatura sobre as fases de ovo e ninfa de $\boldsymbol{D}$. luteipes e $\boldsymbol{D}$. lineare, foram utilizadas câmaras climatizadas reguladas para as temperaturas de $18,20,25,30$ e $32 \pm 1^{\circ} \mathrm{C}$, umidade relativa de $70 \pm 10 \%$ e fotofase de $14 \mathrm{~h}$. Para a fase de ovo, dois casais foram colocados em cada recipiente de criação contendo alimento e quatro canudos que serviram de abrigo e de local para postura (já que os ovos são depositados no interior dos canudos, em local próximo da extremidade que possui algodão umedecido). Vinte e quatro horas após a oviposição, 
as fêmeas e as posturas foram transferidas para as câmaras climatizadas. Foi realizada a contagem do número de ovos e registrada a duração e a viabilidade do período embrionário para 150 ovos em cada temperatura.

Para determinar o efeito da temperatura sobre o estágio ninfal, foram utilizados 25 insetos que eclodiram dos ovos colocados nas temperaturas citadas. Diariamente, foram realizadas observações para avaliar a duração e a viabilidade da fase de ninfa e o número de ínstares em todas as temperaturas. Determinou-se o número de ínstares pela presença de exúvias nos recipientes e/ou pelas características morfológicas das ninfas descritas por ROMEROSUELDO \& DODE (2002), pois algumas ninfas consomem a exúvia após a muda. A duração de cada ínstar foi obtida por meio do registro do intervalo em dias entre as ecdises.

O experimento foi realizado em delineamento inteiramente casualizado, com cinco tratamentos (temperaturas). Os dados foram submetidos à análise de variância, e as médias foram comparadas pelo teste de Tukey $(\mathrm{P}<0,05)$.

A partir dos resultados de duração obtidos para as fases de ovo e de ninfa, determinaram-se o limiar térmico inferior de desenvolvimento $(\mathrm{Tb})$ e a constante térmica $(\mathrm{K})$ pelo método da hipérbole, para D. lineare, e pelo método do coeficiente de variação (CV\%), para D. luteipes, visto que o método da hipérbole é aplicado quando se tem, no mínimo, valores em quatro temperaturas (HADDAD et al., 1999). No caso da determinação pelo método do $\mathrm{CV} \%$, foram arbitrados valores de limiar de desenvolvimento variáveis de $-5 \mathrm{a}+20^{\circ} \mathrm{C}$, a intervalos de 0,1 . A temperatura base foi aquela arbitrada, cujo menor CV (\%) foi encontrado para os valores de $\mathrm{K}\left(\mathrm{K}_{18}, \mathrm{~K}_{20}, \mathrm{~K}_{25}, \mathrm{~K}_{30} \mathrm{e}\right.$ $\mathrm{K}_{32^{\circ} \mathrm{C}}$ ) entre as temperaturas estudadas em laboratório.

\section{RESULTADOS E DISCUSSÃO}

A duração da fase de ovo foi reduzida com a elevação térmica, variando de $17,2\left(18^{\circ} \mathrm{C}\right)$ a $8,0\left(25^{\circ} \mathrm{C}\right)$ dias, para $\boldsymbol{D}$. luteipes, e de $14,5\left(18^{\circ} \mathrm{C}\right)$ a $4,6\left(25^{\circ} \mathrm{C}\right)$ dias, para D. lineare (Tabela 1). REIS et al. (1988) encontraram, para $\boldsymbol{D}$. luteipes, um período de incubação de 7,3 dias a $25^{\circ} \mathrm{C}$. Valor semelhante (7,6 dias), para a mesma espécie, foi obtido por CRUZ et al. (1995).

Em ambas as espécies, observou-se que, na temperatura de $32^{\circ} \mathrm{C}$, não ocorreu eclosão, e que, para D. luteipes, a temperatura de $30^{\circ} \mathrm{C}$ também é prejudicial ao seu desenvolvimento embrionário. Nas demais temperaturas, a viabilidade dos ovos foi superior a $84 \%$, para $\boldsymbol{D}$. luteipes, e para $\boldsymbol{D}$. lineare houve um decréscimo nas temperaturas extremas, e a temperatura de $25^{\circ} \mathrm{C}$ foi a mais favorável (Tabela 1). Semelhante ao observado neste trabalho, LEMOS et al. (1998) também observaram que temperaturas de 15 e $33^{\circ} \mathrm{C}$ desfavorecem o desenvolvimento embrionário de Euborellia annulipes (Lucas) (Dermaptera: Anisolabididae), ou seja, há uma tendência de que as temperaturas na faixa de 18 a $28^{\circ} \mathrm{C}$ sejam as ideais pa ra o desenvolvimento embrionário de dermápteros (SHEPARD et al., 1973; JONES et al., 1988; JAMET \& CAUSSANEL, 1995; LEMOS et al., 1998), o que está de acordo com a faixa de temperatura encontrada para $\boldsymbol{D}$. luteipes e $\boldsymbol{D}$. lineare neste trabalho.

A temperatura promoveu variações na duração dos ínstares para ambas as espécies, com diferenças significativas para o desenvolvimento ninfal. Entretanto, o número de ínstares foi constante (4), independente da temperatura, para ambas as espécies. Para D. luteipes, as durações variaram de 63,6 a 30,4 dias no intervalo de 18 a $25^{\circ} \mathrm{C}$ e, para $\boldsymbol{D}$. lineare, houve uma variação de 60,5 a 20,4 dias, de 18 a $30^{\circ} \mathrm{C}$ (Tabela 2).

Semelhante ao observado, para o período embrionário, as temperaturas de 30 e $32^{\circ} \mathrm{C}$ são prejudiciais ao desenvolvimento das espécies

Tabela 1 - Duração e viabilidade da fase de ovo de Doru luteipes e Doru lineare em diferentes temperaturas. Umidade relativa de $70 \pm 10 \%$ e fotofase de 14 horas.

\begin{tabular}{|c|c|c|c|c|c|c|}
\hline \multirow{3}{*}{ Temperatura $\left({ }^{\circ} \mathrm{C}\right)$} & \multicolumn{3}{|c|}{----------------------------D. luteipes-------------------------- } & \multicolumn{3}{|c|}{---------------------------D. lineare--------------------------- } \\
\hline & \multicolumn{2}{|c|}{------------Duração (dias) ${ }^{3}$------------ } & \multirow{2}{*}{$\begin{array}{c}\text { Viabilidade }^{4} \\
(\% \pm \mathrm{EP})\end{array}$} & \multicolumn{2}{|c|}{--------Duração (dias) ${ }^{5}$-------- } & \multirow{2}{*}{$\begin{array}{l}\text { Viabilidade }^{6} \\
(\% \pm \mathrm{EP})\end{array}$} \\
\hline & Média $^{1} \pm \mathrm{EP}^{2}$ & Intervalo & & Média \pm EP & Intervalo & \\
\hline 18 & $17,2 \pm 0,3 \mathrm{a}$ & $16-19$ & $89,4 \pm 2,2 \mathrm{a}$ & $14,5 \pm 0,2 \mathrm{a}$ & $14-15$ & $48,8 \pm 4,9 b$ \\
\hline 20 & $12,2 \pm 0,5 b$ & $10-14$ & $91,4 \pm 3,9$ a & $10,3 \pm 0,2 b$ & $10-11$ & $59,0 \pm 3,7 b$ \\
\hline 25 & $8,0 \pm 0,2 \mathrm{c}$ & $07-09$ & $84,2 \pm 2,4 \mathrm{a}$ & $6,0 \pm 0,3 \mathrm{c}$ & $05-07$ & $86,6 \pm 3,7 \mathrm{a}$ \\
\hline 30 & & --- & & $4,6 \pm 0,2 \mathrm{~d}$ & $04-05$ & $33,6 \pm 1,0 \mathrm{c}$ \\
\hline 32 & & ---- & & & ---- & \\
\hline
\end{tabular}

${ }^{1}$ Médias seguidas por letras diferentes na coluna diferem significativamente pelo teste de Tukey $(\mathrm{P}=0,05) ;{ }^{2} \mathrm{EP}=\mathrm{Erro}-\mathrm{padrão} ;{ }^{3} \mathrm{~F}=201,89$ e $\mathrm{GL}=2 ;{ }^{4} \mathrm{~F}=1,62$ e $\mathrm{GL}=2 ;{ }^{5} \mathrm{~F}=366,84$ e $\mathrm{GL}=3 ;{ }^{6} \mathrm{~F}=37,9$ e $\mathrm{GL}=3$. 
Tabela 2 - Duração dos ínstares e do período ninfal [Média \pm Erro-padrão (EP)] e viabilidade de ninfas de Doru luteipes e Doru lineare em relação à temperatura. Umidade relativa de $70 \pm 10 \%$ e fotofase de 14 horas.

\begin{tabular}{|c|c|c|c|c|c|c|}
\hline \multirow{2}{*}{ Temperatura $\left({ }^{\circ} \mathrm{C}\right)$} & \multicolumn{5}{|c|}{-------------------------------------------Duração (dias) ------------------------------------------- } & \multirow{2}{*}{$\begin{array}{c}\text { Viabilidade } \\
(\% \pm \mathrm{EP})\end{array}$} \\
\hline & $1^{\circ}$ instar & $2^{\circ}$ instar & $3^{\circ}$ instar & $4^{0}$ instar & Total $^{1}$ & \\
\hline \multicolumn{7}{|c|}{ 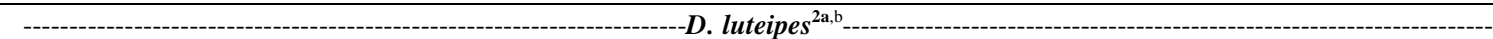 } \\
\hline 18 & $16,7 \pm 0,3$ & $13,2 \pm 0,2$ & $14,4 \pm 0,4$ & $19,2 \pm 0,39$ & $63,6 \pm 0,1$ a & $93,0 \pm 0,8$ a \\
\hline 20 & $12,8 \pm 0,2$ & $8,7 \pm 0,1$ & $8,8 \pm 0,41$ & $13,2 \pm 0,1$ & $43,5 \pm 0,1 b$ & $85,7 \pm 0,7 b$ \\
\hline 25 & $8,2 \pm 0,2$ & $5,7 \pm 0,3$ & $7,3 \pm 0,65$ & $9,2 \pm 0,2$ & $30,4 \pm 0,2 \mathrm{c}$ & $86,9 \pm 0,9 b$ \\
\hline 30 & --- & --- & --- & --- & --- & --- \\
\hline 32 & --- & --- & --- & --- & --- & --- \\
\hline 18 & $16,4 \pm 0,5$ & $12,9 \pm 0,3$ & $13,1 \pm 0,2$ & $18,0 \pm 0,2$ & $60,5 \pm 0,6 \mathrm{a}$ & $39,4 \pm 1,1 d$ \\
\hline 20 & $11,1 \pm 0,2$ & $8,1 \pm 0,2$ & $8,3 \pm 0,2$ & $11,0 \pm 0,2$ & $38,5 \pm 0,34 b$ & $60,0 \pm 0,8 b$ \\
\hline 25 & $6,4 \pm 0,2$ & $4,6 \pm 0,2$ & $5,1 \pm 0,2$ & $7,3 \pm 0,2$ & $23,5 \pm 0,48 \mathrm{c}$ & $77,1 \pm 0,9 \mathrm{a}$ \\
\hline 30 & $5,6 \pm 0,3$ & $4,1 \pm 0,1$ & $4,8 \pm 0,2$ & $5,8 \pm 0,2$ & $20,4 \pm 0,5 d$ & $46,4 \pm 1,1 \mathrm{c}$ \\
\hline 32 & --- & --- & --- & --- & --- & --- \\
\hline
\end{tabular}

${ }^{1}$ Médias seguidas por letras diferentes na coluna diferem significativamente pelo teste de Tukey $(\mathrm{P}<0,05) ;{ }^{2 a} \mathrm{~F}(\mathrm{Total})=907,29$ e $\mathrm{GL}=2 ;{ }^{2 b}$ $\mathrm{F}($ Viabilidade $)=22,44$ e $\mathrm{GL}=2 ;{ }^{3 \mathrm{a}} \mathrm{F}($ Total $)=1574,62$ e $\mathrm{GL}=3 ;{ }^{3 \mathrm{a}} \mathrm{F}$ (Viabilidade $)=279,69$ e $\mathrm{GL}=3$.

estudadas, sendo mais drástica a influência para $\boldsymbol{D}$. luteipes, uma vez que não ocorreu emergência a partir de $30^{\circ} \mathrm{C}$. A temperatura exerceu maior efeito sobre a viabilidade ninfal em $\boldsymbol{D}$. lineare, sendo observados valores superiores na faixa de 20 a $25^{\circ} \mathrm{C}$, decrescendo a 18 e $30^{\circ} \mathrm{C}$. Para $\boldsymbol{D}$. luteipes, dentro da faixa de temperatura em que ocorreu o desenvolvimento, emergindo adultos, registraram-se viabilidades superiores a $85 \%$, havendo diferença significativa apenas na temperatura de $18^{\circ} \mathrm{C}$ (Tabela 2).

Para as duas espécies de dermápteros, o tempo de desenvolvimento (ovo-adulto) foi inversamente proporcional à temperatura, variando de $80,8 \operatorname{dias}\left(18^{\circ} \mathrm{C}\right)$ a $38,4 \operatorname{dias}\left(25^{\circ} \mathrm{C}\right)$ e de $75 \operatorname{dias}\left(18^{\circ} \mathrm{C}\right)$ a 25 dias $\left(30^{\circ} \mathrm{C}\right)$, para $D$. luteipes e $D$. lineare, respectivamente (Figura $1 \mathrm{~A} \mathrm{e} \mathrm{B})$. A viabilidade desse período variou de $83,1 \%\left(18^{\circ} \mathrm{C}\right)$ até $73,1 \%\left(25^{\circ} \mathrm{C}\right)$ para D. luteipes, decrescendo, portanto, com a elevação da temperatura (Figura 1A). Para D. lineare, a viabilidade total máxima foi a $25^{\circ} \mathrm{C}$ e não atingiu $70 \%$, antecedida por 35,4, 19,2 e 15,6\% nas temperaturas de 20, 18 e $30^{\circ} \mathrm{C}$, respectivamente, indicando que, na faixa térmica entre 18 e $30^{\circ} \mathrm{C}, \boldsymbol{D}$. luteipes é menos sensível à temperatura que $\boldsymbol{D}$. lineare (Figura $1 \mathrm{Ae} \mathrm{B}$ ).

Para as fases de ovo, ninfa e ciclo biológico (ovo-adulto) de $\boldsymbol{D}$. luteipes, determinou-se uma temperatura base (Tb) de 11,6; 10,9 e $11,0^{\circ} \mathrm{C}$, respectivamente, com constates térmicas de 107,7; 424,0 e 529,0GD, respectivamente. O coeficiente de determinação foi superior a 0,960 , acima do preconizado pelo método da hipérbole $(0,900)$ (Tabela 3). Para $\boldsymbol{D}$. lineare, a Tb foi de 12,$2 ; 10,8$ e $11,1^{\circ} \mathrm{C}$, sendo a constante térmica de 80,2; 370,4 e 450,4GD, respectivamente, para as fases de ovo, ninfa e período ovo-adulto (Tabela 3). Os valores estimados de Tb caracterizam as espécies como de clima subtropical, já que os valores ficaram próximos de $10,5^{\circ} \mathrm{C}$, preconizado por HONÉK (1996), embora as espécies tenham uma ampla distribuição no território nacional.

Ainda que as espécies estudadas tenham uma ampla distribuição no território nacional, a diferença nas exigências térmicas pode determinar variações no padrão de distribuição geográfica dessas espécies, possibilitando a ação destas no controle integrado de pragas do milho em diferentes regiões agrícolas do país. Dados preliminares de coletas realizadas em plantações de milho em Tangará da Serra, região Sudoeste do Estado de Mato Grosso, cujas temperaturas variam entre 16 e $36^{\circ} \mathrm{C}$, demonstraram a predominância significativa de $\boldsymbol{D}$. lineare (Dados não publicados), reforçando as diferenças nas exigências térmicas encontradas para as duas espécies estudadas.

Cabe salientar que, embora $\boldsymbol{D}$. lineare tenha se desenvolvido em uma faixa maior de temperatura (de 18 a $30^{\circ} \mathrm{C}$ ) em relação a $\boldsymbol{D}$. luteipes (de 18 a $25^{\circ} \mathrm{C}$ ), esta última apresentou, nessas temperaturas, taxas de sobrevivência de ovos e ninfas superiores a $84 \%$, enquanto que, para $\boldsymbol{D}$. lineare, as viabilidades máximas registradas foram a $25^{\circ} \mathrm{C}$, sendo de $86,6 \%$ e $77,1 \%$, para ovos e ninfas, respectivamente (Tabela 2). 


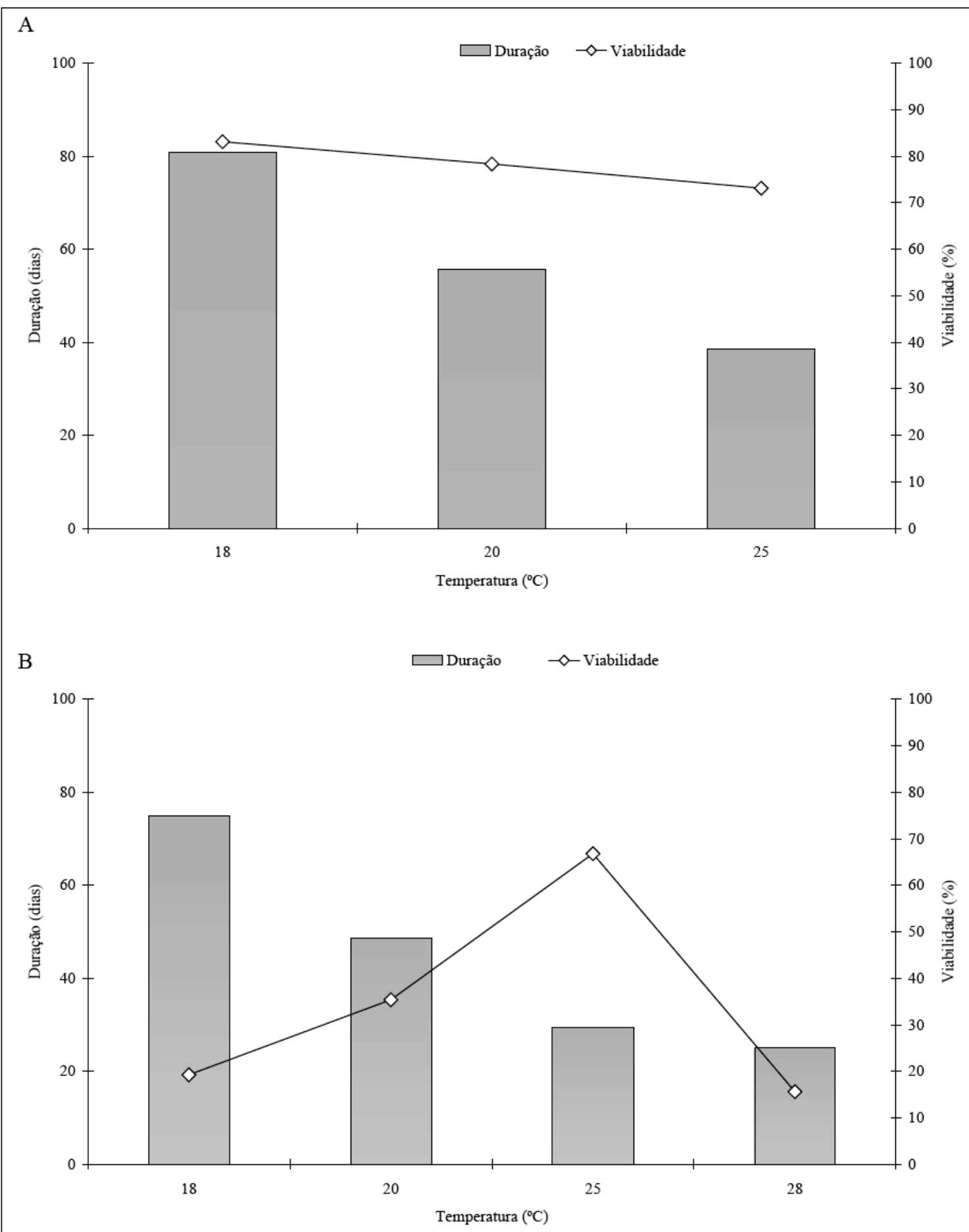

Figura 1 - Duração média e viabilidade do ciclo biológico (ovo-adulto) de $\boldsymbol{D}$. luteipes (A) e $\boldsymbol{D}$. lineare (B) em diferentes temperaturas. Umidade relativa de $70 \pm 10 \%$ e fotofase de 14 horas.

\section{CONCLUSÃO}

A espécie de tesourinha Doru lineare apresenta ciclo de desenvolvimento mais curto, portanto com possibilidades de apresentar um maior número de gerações/ano em relação à espécie $\boldsymbol{D}$. luteipes. Tanto $D$. luteipes e $D$. lineare não se desenvolvem na temperatura constante de $32^{\circ} \mathrm{C}$. Dentre as temperaturas avaliadas, a mais adequada para a criação de $\boldsymbol{D}$. luteipes e $\boldsymbol{D}$. lineare foi de $25^{\circ} \mathrm{C}$. 
Tabela 3 - Temperatura base (Tb), constante térmica (K), equação da velocidade de desenvolvimento (1/D) e coeficiente de determinação $\left(\mathrm{R}^{2}\right)$ das diferentes fases de desenvolvimento e do ciclo biológico (ovo-adulto) de Doru luteipes e Doru lineare.

\begin{tabular}{|c|c|c|c|c|c|}
\hline Fase & Espécie & $\mathrm{Tb}\left({ }^{\circ} \mathrm{C}\right)$ & $\mathrm{K}(\mathrm{GD})$ & Equação (1/D) & $\mathrm{R}^{2}(\%)$ \\
\hline \multicolumn{6}{|c|}{ D. luteipes $^{1}$} \\
\hline Ovo & & 11,57 & 107 & ---- & ---- \\
\hline Ninfa & & 10,9 & 424 & ---- & ---- \\
\hline Ciclo biológico & & 11,03 & 529 & ---- & ---- \\
\hline \multicolumn{6}{|c|}{ D. lineare ${ }^{2}$} \\
\hline Ovo & & 12,21 & 80,2 & $Y=0,01247 x-0,15231$ & 0,99 \\
\hline Ninfa & & 10,83 & 370,4 & $Y=0,0027 x-0,02924$ & 0,99 \\
\hline Ciclo biológico & & 11,14 & 450,4 & $Y=0,00222 x-0,02472$ & 0,96 \\
\hline
\end{tabular}

${ }^{1}$ Método do CV(\%); ${ }^{2}$ Método da Hipérbole.

\section{AGRADECIMENTOS}

À Dra. Marinéia de Lara Haddad, pelo auxílio na determinação das exigências térmicas.

\section{REFERÊNCIAS}

ALVARENGA, C.D. et al. Biologia e predação de Doru luteipes (Scudder) sobre Schizaphis graminum (Rondani) criado em diferentes genótipos de sorgo. Anais da Sociedade Entomológica do Brasil, v.24, p.523-531, 1995a.

ALVARENGA, C.D. et al. Controle integrado de Schizaphis graminum (Rondani) em sorgo através de genótipos resistentes e do predador Doru luteipes (Scudder). Anais da Sociedade Entomologica do Brasil, v.24, n.3, p.507-516, 1995 b.

ALVARENGA, C.D. et al. Efeito do predador Doru luteipes (Scudder) sobre o crescimento populacional de Schizaphis graminum (Rondani) em diferentes genótipos de sorgo. Anais da Sociedade Entomológica do Brasil, v.25, p.137-140, 1996.

BERTI FILHO, E.; CIOCIOLA, A.I. Parasitóides ou predadores? Vantagens e desvantagens. In: PARRA, J.R.P. et al. Controle biológico no Brasil. São Paulo: Manole, 2002. Cap.2, p.2941 .

CÔNSOLI, F.I; PARRA, J.R.P. Produção in vitro de parasitóides: criação de Trichogramma galoi e T. pretiosum no Brasil. In: PARRA, J.R.P.; ZUCCHI, R.A. Trichogramma e o controle biológico aplicado. Piracicaba: FEALQ, 1997. p.121-150.

CRUZ, I. Potencial de Doru luteipes como predador de Spodoptera frugiperda em condições de campo. Sete Lagoas: CNPMS, 1991. V.4, p.85-86. (Relatório Técnico Anual do Centro Nacional de Pesquisa de Milho e Sorgo 1985/1987).

CRUZ, I. Aplicação de inseticidas para o controle da lagarta-do-cartucho Spodoptera frugiperda, e sua ação sobre o inimigo natural Doru luteipes. Sete Lagoas: CNPMS, 1994. V.6, 82p. (Relatório Técnico Anual do Centro Nacional de Pesquisa de Milho e Sorgo 1992/1993).
CRUZ, I. Controle biológico de pragas na cultura de milho para produção de conservas (Minimilho), por meio de parasitóides e predadores. Sete Lagoas: CNPMS, 2007. V.91, 16p. (Circular Técnica, Embrapa Milho e Sorgo).

CRUZ, I. et al. Biologia de Doru luteipes (Scudder) e sua capacidade predatória de ovos de Helicoverpa zea (Boddie). Anais da Sociedade Entomológica do Brasil, v.24, p.273278, 1995.

FERNANDES, O.D. Efeito do milho geneticamente modificada (MON810) em Spodoptera frugiperda (J.E. Smith, 1797) e no parasitóide de ovos Trichogramma spp. 2003. 182f. Tese (Doutorado em Entomologia) - Escola Superior de Agricultura "Luiz de Queiroz”, Universidade de São Paulo, SP.

FIGUEIREDO, M.L.C. et al. Associação entre inimigos naturais e Spodoptera frugiperda (J.E. Smith, 1797) (Lepidoptera: Noctuidae) na cultura do milho. Revista Brasileira de Milho e Sorgo, v.5, n.3, p.340-350, 2006.

GASSEN, D.N. Parasitos, patógenos e predadores de insetos associados à cultura do trigo. Passo Fundo: EMBRAPA/CNPT, $1986.86 p$

GUERREIRO, J.C. et al. Ocorrência estacional de Doru luteipes na cultura do milho em São Paulo, Brasil. Manejo Integrado de Plagas \& Agroecologia, v.70, p.46-49, 2003.

HADDAD, M.L. et al. Métodos para estimar os limites térmicos inferior e superior de desenvolvimento de insetos. São Paulo: Fealq, 1999. 29p.

HAGEN, K.S. et al. The biology and impact of predators. In: HUFFAKER, C.B.; MESSENGER, P.S. Theory and practice of biological control. New York: Academic, 1976. p.93142 .

HONÉK, A. Geographical variation in thermal requirements for insect development. European Journal of Entomology, v.93, p.303-312, 1996.

JAMET, C.; CAUSSANEL, C. Données biologiques, fonctionnement des apareils génitaux, comportements sexuels et maternels chez Euborellia annulipes (Lucas) (Demaptère, 
Carcinophoridae). Bulletin de la Société Entomologique de France, v.100, p.37-58, 1995.

JONES, R.W. et al. Biology and life tables for the predaceous earwig, Doru taeniatum (Dermaptera: Forficulidae). Entomophaga, v.33, n.1, p.43-54, 1988.

LEITE, L.G; LARA, F.M. Flutuação populacional de insetos e inimigos naturais associados à cultura da soja em Jaboticabal, SP. Anais da Sociedade Entomológica do Brasil, v.14, p.45-57, 1985.

LEMOS, W.P. et al. Influência da temperatura no desenvolvimento de Euborellia annulipes (Lucas) (Dermaptera: Anisolabididade), predador do bicudo-do-algodoeiro. Anais da Sociedade Entomológica do Brasil, v.27, n.1, p.67-76, 1998. Disponível em: <http://www.scielo.br/pdf/aseb/v27n1/v27n1a09.pdf>. Acesso em: 08 jun. 2010. doi: 10.1590/S0301-80591998000100009.

NAVA, D.E. et al. Exigências térmicas, estimativa do número de gerações de Stenoma catenifer e comprovação do modelo em campo. Pesquisa Agropecuária Brasileira, v.40, p.961967, 2005. Disponível em: <http://www.scielo.br/pdf/pab/ v40n10/a03v4010.pdf>. Acesso em: 08 jun. 2010. doi: 10.1590/S0100-204X2005001000003.

PARRA, J.R.P. Técnicas de criação de Anagasta kuehniella, hospedeiro alternativo para produção de Trichogramma. In: PARRA, J.R.P.; ZUCCHI, R.A. Trichogramma e o controle aplicado. Piracicaba, FEALQ, 1997. p.121-150.

PASINI A. et al. Dieta artificial para criação de Doru luteipes (Scudder) (Dermaptera: Forficulidae), predador da lagarta do cartucho do milho, Spodoptera frugiperda (J.E. Smith) (Lepidoptera: Noctuidae). Neotropical Entomology, v.36, n.2, p.308-311, 2007. Disponível em: <http://www.scielo.br/ pdf/ne/v36n2/a20v36n2.pdf $>$. Acesso em: 08 jun. 2010. doi: 10.1590/S1519-566X2007000200020.

PICANÇO, M.C. et al. Seletividade de inseticidas a D. luteipes (Scudder, 1876) (Dermaptera: Forficulidae) e Cotesia sp. (Hymenoptera: Braconidae) inimigos naturais de Ascia monuste orseis (Godart, 1818) (Lepidoptera: Pieridae). Ciência Rural, v.33, p.183-188, 2003. Disponível em: <http://www.scielo.br/ pdf/cr/v33n2/15203.pdf $>$. Acesso em: 08 jun. 2010. doi: 10.1590/S0103-84782003000200001.
PRATISSOLI, D.; PARRA, J.R.P. Desenvolvimento e exigências térmicas de Trichogramma pretiossum Riley, criados em duas traças do tomateiro. Pesquisa Agropecuária Brasileira, v.35, p.1284-1288, 2000. Disponível em: <http://www.scielo.br/ pdf/pab/v35n7/1281.pdf $>$. Acesso em: 08 jun. 2010. doi: 10.1590/S0100-204X2000000700001.

REIS, L.L. et al. Biologia e potencial de Doru luteipes no controle de Spodoptera frugiperda. Pesquisa Agropecuária Brasileira, v.23, p.333-342, 1988.

ROMERO-SUELDO, M.; CUEZZO, F. Presencia de Paratrechina silvestri (Hymenoptera: Formicidae) posible enemigo natural de Doru lineare (Dermaptera: Forficulidae) em cultivos de maiz. Neotropica, v.47, p.60, 2001.

ROMERO-SUELDO, M.; DODE, M. Description de los estados inmaduros de Doru lineare (Dermaptera: Forficulidae) y de su ciclo de vida en maiz en Tucuman (Argentina). Acta Zoologica Lilloana, v.46, n.1, p.71-80, 2002.

SHEPARD, M. et al. Biology of the predaceous earwig Labidura riparia (Dermaptera: Labiduridae). Annals of the Entomological Society of America, v.66, p.837-841, 1973.

SIMÕES, J.C. et al. Seletividade de inseticidas às diferentes fases de desenvolvimento do predador Doru luteipes (Scudder) (Dermaptera: Forficulidae). Anais da Sociedade Entomológica do Brasil, v.27, p.289-294, 1998. Disponível em: <http://www.scielo.br/pdf/aseb/v27n2/v27n2a16.pdf>. Acesso em: 08 jun. 2010. doi: 10.1590/S030180591998000200016 .

SOARES, J.J. et al. Impacto de herbicidas sobre artrópodos benéficos associados ao algodoeiro. Pesquisa Agropecuária Brasileira, v.30, p.1135-1140, 1995.

SOARES, J.J.; BUSOLI, A.C. Efeito de inseticidas em insetos predadores em culturas de algodão. Pesquisa Agropecuária Brasileira, v.35, p.1889-1894, 2000. Disponível em: <http:/ /www.scielo.br/pdf/pab/v35n9/v35n9a23.pdf>. Acesso em: 08 jun. 2010. doi: 10.1590/S0100-204X2000000900023.

VIANA, P.A. Ocorrência e controle de pragas na safrinha de milho nas regiões Norte e Oeste do Paraná. Sete Lagoas: CNPMS, 2004. 12p. (Circular Técnica, 45). 